\title{
Critical phenomena in atmospheric precipitation
}

\author{
OLE PETERS ${ }^{1,2,3 *}$ AND J. DAVID NEELIN ${ }^{3}$ \\ ${ }^{1}$ Santa Fe Institute, 1399 Hyde Park Road, Santa Fe, New Mexico 87501, USA \\ ${ }^{2}$ CNLS, Los Alamos National Laboratory, MS-B258, Los Alamos, New Mexico 87545, USA \\ ${ }^{3}$ Department of Atmospheric Sciences and Institute of Geophysics and Planetary Physics, University of California, Los Angeles, 405 Hilgard Ave., Los Angeles, \\ California 90095-1565, USA \\ *e-mail: ole.peters@physics.org
}

C ritical phenomena occur near continuous phase transitions. As a tuning parameter crosses its critical value, an order parameter increases as a power law. At criticality, order-parameter fluctuations diverge and their spatial correlation decays as a power law ${ }^{1}$. In systems where the tuning parameter and order parameter are coupled, the critical point can become an attractor, and self-organized criticality (SOC) results ${ }^{2,3}$. Here we argue, using satellite data, that a critical value of water vapour (the tuning parameter) marks a non-equilibrium continuous phase transition to a regime of strong atmospheric convection and precipitation (the order parameter)-with correlated regions on scales of tens to hundreds of kilometres. Despite the complexity of atmospheric dynamics, we find that important observables conform to the simple functional forms predicted by the theory of critical phenomena. In meteorology the term 'quasi-equilibrium' refers to a balance between slow large-scale driving processes and rapid release of buoyancy by moist convection ${ }^{4}$. Our study indicates that the attractive quasi-equilibrium state, postulated long before SOC (ref. 5), is the critical point of a continuous phase transition and is thus an instance of SOC.

At short timescales the majority of tropical rainfall occurs in intense events with rain rates exceeding the climatological mean by an order of magnitude or more. Moist convection and the accompanying precipitation have been found to be sensitive to variations in water vapour along the vertical on large space and timescales both in observations $s^{6,7}$ and in models ${ }^{8-10}$. This is due to the effect of water vapour on the buoyancy of cloud plumes as they entrain surrounding air by turbulent mixing. We conjecture that the transition to intense convection, accompanying the onset of intense precipitation, shows signs of a continuous phase transition. Note that such a large-scale continuous phase transition involving the flow regime of the convecting fluid is entirely different from the well-known discontinuous phase transition of condensation at the droplet scale. We analysed satellite microwave estimates of rainfall rate, $P$, water vapour, $w$, cloud liquid water and sea surface temperature (SST) from the Tropical Rainfall Measuring Mission from 2000 to 2005. Observations from the western Pacific

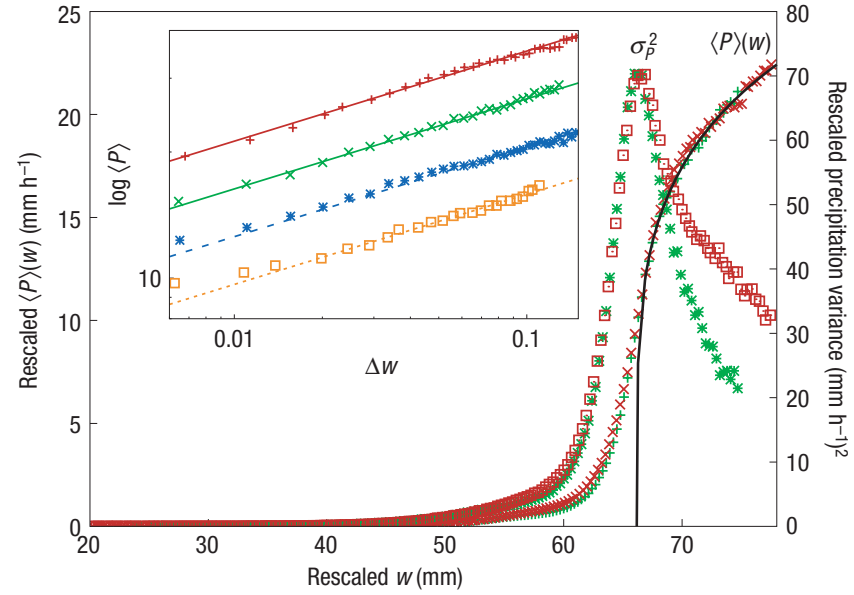

Figure 1 Order parameter and susceptibility. The collapsed (see text) precipitation rates $\langle P\rangle(w)$ and their variances $\sigma_{P}^{2}(w)$ for the tropical eastern (red) and western (green) Pacific as well as a power-law fit above the critical point (solid line). The inset shows on double-logarithmic scales the precipitation rate as a function of reduced water vapour (see text) for western Pacific (green, 120E to 170W), eastern Pacific (red, 170W to 70W), Atlantic (blue, 70W to 20E), and Indian 0cean (pink, 30E to $120 \mathrm{E}$ ). The data are shifted by a small arbitrary factor for visual ease. The straight lines are to guide the eye. They all have a slope of 0.215 , fitting the data from all regions well.

provided initial support for our conjecture: a power-law pick-up of precipitation above a critical value, $w_{c}$, of water vapour was observed. We proceeded to test whether other observables also behaved as predicted by the theory of phase transitions.

To motivate our conjecture in terms of the current understanding of SOC, consider a generic lattice-based model. Particle-conserving rules defining the model ascribe a number of particles to every lattice site, and demand hopping of particles to 


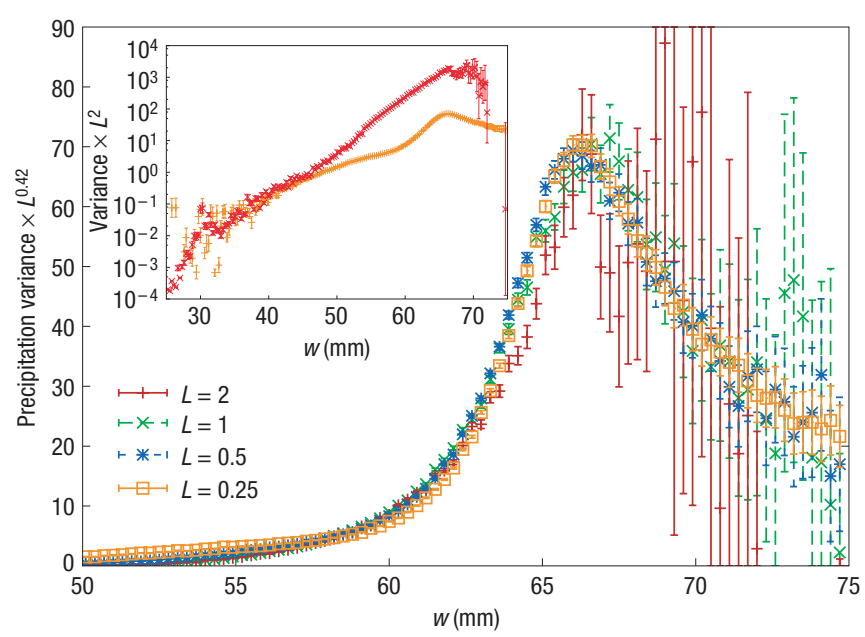

Figure 2 Finite-size scaling. The variance of the order parameter $\sigma_{P}^{2}(w)$ as a function of $w$, rescaled with $L^{0.42}$ for system sizes $0.25^{\circ}, 0.5^{\circ}, 1^{\circ}$ and $2^{\circ}$ in the western Pacific. From $w \approx 57 \mathrm{~mm}$, this produces a good collapse. The inset shows that away from the critical point, up to $w \approx 40 \mathrm{~mm}$ a trivial rescaling with $L^{d=2}$ works adequately. This suggests that the non-trivial collapse is indeed a result of criticality. The error bars are standard errors, determined through the zeroth, second and fourth moments of the distribution of $P$ at any given $w$. Measurements of $P(w)$ are considered independent, which holds well between satellite overpasses, although not within individual tracks.

nearest-neighbour sites when a local density threshold is exceeded. The global effect of these rules is a phase transition at a critical value of the global particle density between a quiescent phase (where the system eventually settles into a stable configuration) and an active phase (where stable configurations are inaccessible). The tuning parameter is the particle density and the order parameter is identified as the density of active sites ${ }^{11}$. SOC can be described in terms of such absorbing-state phase transitions ${ }^{11,12}$. Here a coupling between order parameter and tuning parameter is introduced by opening the boundaries and adding a slow drive: whenever activity ceases, a new particle is added to the system, that is, an increase in the tuning parameter. On the other hand, large activity leads to dissipation (particle loss) at the boundaries, that is, a reduction of the tuning parameter. Such open, slowly driven systems organize themselves to the critical point of the corresponding (closed boundaries, no drive) absorbing-state phase transition. The scale-free avalanche size distributions in SOC models result from the proximity of the system to a critical point. Physical SOC systems are typically investigated at criticality. This prohibits the characterization of physical SOC systems in terms of exponents defined in the approach to criticality. Also in silico studies of the equivalence of absorbing-state and SOC models ${ }^{13}$ are still rare, and fundamental questions remain open ${ }^{14}$.

From the meteorological perspective, our conjecture is motivated as follows. Atmospheric convection has long been viewed in terms of a slow drive (surface heating and evaporation) and fast dissipation/loss processes (of buoyancy and rainwater) in precipitating convection. Surface heating and evaporation drive turbulent mixing that maintains a moist atmospheric boundary layer. Combined with radiative cooling, conditional instability is created. Whereas sub-saturated air remains stable, saturated condensing plumes can rise through the full depth of the tropical troposphere. The fast dissipation by moist convection prevents the troposphere from deviating strongly from marginal stability ${ }^{15}$.
Although observational tests of this approximate quasi-equilibrium (QE) state of the tropical troposphere have limited precision, it forms the basis of most convective parametrizations in large-scale models ${ }^{16}$ and much tropical dynamical theory ${ }^{17,18}$. Taking large-scale flows into account modifies the process in space and time but does not change it fundamentally. This perspective suggests that a critical point in the water vapour would act as an attractor. Indeed this is basically the convective QE postulate ${ }^{4}$.

The critical value $w_{c}$ depends, for example, on atmospheric temperature, but for present purposes this translates well enough into a critical amount of water vapour for a given climatic region within the tropics. Regions here are defined by longitude ranges given in the caption of Fig. 1 corresponding to major ocean basins, for oceanic grid-points within $20 \mathrm{~S}-20 \mathrm{~N}$. Data are collected at $0.25^{\circ}$ latitude-longitude resolution and are effectively snapshots in time. The observable $w$ captures vertically integrated, or column, water vapour (given as liquid-water-equivalent volume per area, in $\mathrm{mm}$ ). In the following, \langle\rangle refers to an average conditioned on water vapour over all observations in a given region over the investigated 5-year period.

In Fig. 1 we show, as a function of the tuning parameter $w$, the average value of the order parameter $\langle P\rangle(w)$ and the susceptibility of the system, represented by the order parameter variance, $\sigma_{P}^{2}(w)$, discussed following equation (2). The ensemble size for the average ranges from a few thousand at extremes to $10^{6}$ at typical $w$ values. Above $w_{c}$, the order parameter is well approximated by the standard form ${ }^{1}$

$$
\langle P\rangle(w)=a\left(w-w_{\mathrm{c}}\right)^{\beta}
$$

where $a$ is a system-dependent constant and $\beta$ is a universal exponent. The deviations from power-law behaviour below $w_{\mathrm{c}}$ in Fig. 1 are typical of critical systems of finite size ${ }^{19}$.

The critical value $w_{c}$ is non-universal and changes with regional climatic conditions, as does the amplitude $a$. To test the degree to which curves from different regions $i$ collapse, we rescaled the $w$ values in Fig. 1 by factors $f_{w}^{i}$, reflecting the non-universality of $w_{c}$ and $\langle P\rangle(w)$ and $\sigma_{P}^{2}(w)$ by $f_{P}^{i}$ and $f_{\sigma^{2}}^{i}$, respectively (setting western Pacific factors to 1$)$. For visual clarity, the data collapse in Fig. 1 is shown only for the eastern and western Pacific-climatically very different regions. Similar agreement occurs for other regions (steps in the rescaling and figures for all regions are provided in the Supplementary Information). The exponent $\beta$ seems to be universal and independent of the climatic region. In the inset of Fig. 1 we show the average precipitation as a function of the reduced water vapour $\Delta w \equiv\left(w-w_{\mathrm{c}}\right) / w_{\mathrm{c}}$ in a double-logarithmic plot. Importantly, power laws fitted to these distributions all have the same exponent (slope) to within \pm 0.02 . The data points in Fig. 1 represent the entire observational period, including all observed SSTs. Conditioning averages by SST ranges yields similar results (see Fig. 3 and the Supplementary Information), slightly reducing the sub-critical part of the curves.

We define the susceptibility $\chi(w ; L)$ by means of the variance of the order parameter $\sigma_{P}^{2}$ :

$$
\chi(w ; L)=L^{d} \sigma_{P}^{2}(w ; L)
$$

where $d$ denotes the dimensionality of the system and $L$ denotes the spatial resolution. Figure 1 shows a suggestive increase in $\sigma_{P}^{2}$ near $w_{\mathrm{c}}$, and indicates that standard methods for critical phenomena can sensibly be applied.

Next we test for finite-size scaling. Because our system size cannot be changed, we identify the spatial data resolution $L$ as the relevant length scale. Changing $L$ has the effect of taking averages over different numbers of degrees of freedom and allows us to 


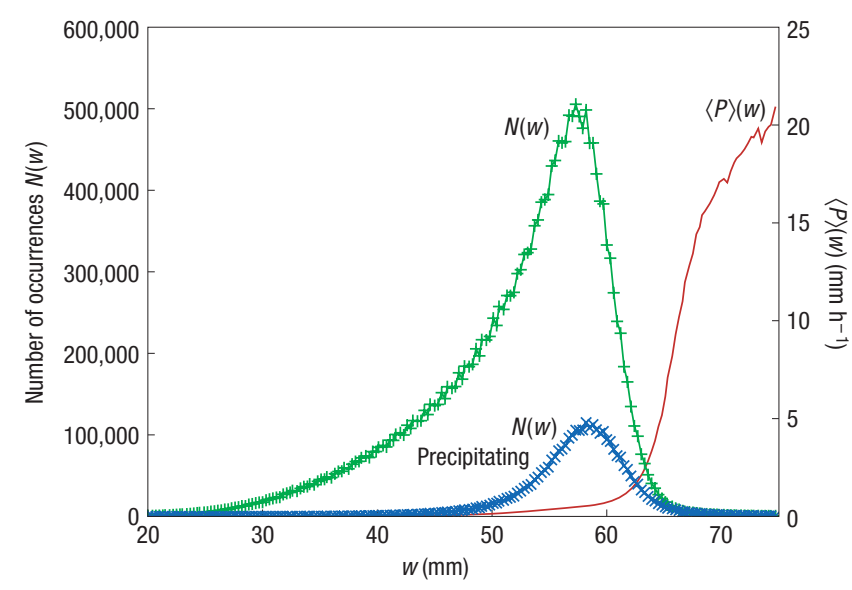

Figure 3 Residence times. The number of times $N(w)$ an atmospheric pixel of $0.25^{\circ} \times 0.25^{\circ}$ was observed at water vapour $w$ in the western Pacific, given an SST within a $1^{\circ} \mathrm{C}$ bin at $30^{\circ} \mathrm{C}$. The green and blue lines show residence time for all points and precipitating points, respectively. The red line shows the order-parameter pick-up $\langle P\rangle(w)$ for orientation (precipitation scale on the right).

investigate the degree of spatial correlation. The finite-size scaling ansatz for the susceptibility is

$$
\chi(w ; L)=L^{\gamma / v} \tilde{\chi}\left(\Delta w L^{1 / v}\right),
$$

defining $\gamma$ and $v$ as the standard critical exponents and the usual finite-size scaling function $\tilde{\chi}(x)$, constant for small arguments $|x| \ll 1$ and decaying as $|x|^{-\gamma}$ for large arguments $|x| \gg 1$ (ref. 20). The variance $\sigma_{P}^{2}(w ; L)$ is affected by uncertainties in $w$ and $w_{c}$, making precise quantification of $\chi(w ; L)$ difficult. Therefore, we do not estimate $\gamma$ from the $w$-dependence of $\chi(w ; L)$, corresponding to large arguments $|x|$ in equation (3) but effectively fix $\Delta w=$ $0=x$ and obtain $\gamma / v$ from the $L$-dependence of the maximum susceptibility $\chi^{\max }(L)$.

The variance of the average $\langle P\rangle(w ; L)$ over $L^{d}$ independent degrees of freedom decreases as $\sigma_{P}^{2}(w ; L) \propto L^{-d}$. In a critical system, however, the diverging bulk correlation length $\xi \propto(\Delta w)^{-v} \gg$ $L$ (small argument in equation (3)) prohibits the assumption of independence. In this case equation (3) with (2) yields

$$
\sigma_{P}^{2 \max }(L) \propto L^{\gamma / \nu-d} .
$$

Coarsening the spatial resolution of the data, we find in Fig. 2 that $\sigma_{P}^{2 \max }(L)$ scales roughly as $L^{-\lambda}$, with $\lambda=0.46(4)$. This suggests the exponent ratio $\gamma / v=1.54(4)$.

At criticality, the spatial decay of correlations between order-parameter fluctuations becomes scale-free ${ }^{1}$. This is equivalent to a non-trivial power-law dependence of the order-parameter variance on $L$ (see the Supplementary Information for details and conditions). Hence, Fig. 2 indicates a scale-free correlation function of fluctuations in the rain rate in the range of $25-200 \mathrm{~km}$. This suggests that the meteorological features known as mesoscale convective systems ${ }^{21}$ are long-range correlation structures akin to critical clusters ${ }^{22}$. Synoptic inspection indicates that the high rain-rate phase and critical region of Fig. 1 come substantially from points within such complexes (examples are provided in the Supplementary Information).

The question of self-organization towards the critical point of the transition is addressed by showing the residence times of the system in Fig. 3. This is the number of observations in the 5-year period where the system was found at a given level of water vapour. A slowly driven system would be expected to spend a significant amount of time in the low- $w$ phase because when it fluctuates into this phase, for example, owing to some large-scale event, it takes a long time to recover. Therefore, the distribution decreases slowly towards low values of $w$. On the other hand, the fast dissipation mechanism ensures that the system leaves the high- $w$ regime relatively quickly when it fluctuates into it. Consequently, the distribution decreases rapidly towards large values of $w$. For the properties of rainfall, the part of the distribution in Fig. 3 comprised only of observations with rainfall is of interest, seen as the blue line in Fig. 3. We note that the system has the highest probability of being found near the beginning of the intense precipitation regime. Almost the entire weight of the distribution of rainy times is concentrated here.

Meteorologically, these results suggest a means to redefine and extend convective QE, both empirically and theoretically. In its simplest application, QE assumes that the relationship among atmospheric column thermodynamic variables is pinned close to the point where deep convection and precipitation set in. Figure 3 shows this to be a reasonable first approximation, but it also implies associated critical phenomena. A loss term $\langle P\rangle(w)$ of the form of equation (1) implies the absence of a well-defined convective timescale. Scale-free distributions of event $\operatorname{sizes}^{23}$ and the spatial correlation behaviour seen in Fig. 2 may result from this proximity to an apparent continuous phase transition. These properties will be important to include in stochastic convective parametrizations that extend QE to include fluctuations ${ }^{24}$ and in understanding the relationship of QE to mesoscale variability.

These findings beg for a simple model of the atmospheric dynamics responsible for the critical behaviour. Although the physics must conform with recent cloud-resolving model analysis of mesoscale aggregation ${ }^{8,25}$, our results point to the key role of excitatory short-range interactions, essential for critical phenomena of the type seen here. SOC has been proposed as an explanation for scale-free behaviour in many different physical systems ${ }^{26}$. Attention has been focused on avalanche size distributions, partly due to the difficulty of measuring standard observables for critical phenomena, such as order parameters, tuning parameters, or susceptibilities. The present study advances our understanding of SOC by identifying these observables in a physical system in which both the underlying phase transition and the tendency of the system to reside near the critical point can be demonstrated.

\section{METHODS}

Data are from the TMI (TRMM microwave imager), processed by Remote Sensing Systems. The spatial resolution reflects the footprint of the instrument. As with any satellite retrieval product, it is necessary to consider whether the algorithm assumptions could impact the results. The microwave retrieval algorithm is that used on special sensor microwave imager (SSM/I) data ${ }^{27}$. The combination of four microwave channels permits independent retrieval of water vapour and condensed phase water (with SST and surface wind speed), whereas an empirical relation is used to partition cloud water and rain. Column water vapour validates well against in situ sounding data, which also show that daily variations are largely associated with the lower troposphere above the atmospheric boundary layer ${ }^{6}$. Validation of TMI rain rate against space-borne precipitation radar at sub-daily timescales in the tropical western Pacific ${ }^{28}$ show TMI overestimating rain rate but with an approximately linear relationship to precipitation radar. The results here are insensitive to linear scaling. We have carried out a number of checks to verify that results are not substantially impacted by a high rain rate cutoff in the algorithm $\left(25 \mathrm{~mm} \mathrm{~h}^{-1}\right)$, including comparison with regions where cutoff occurrences are very low, such as the eastern Pacific (Fig. 1). The clearest check is that the essential features are identical for the cloud liquid water, whose measurement cutoff of $2.5 \mathrm{~mm}$ is 
never reached (see the Supplementary Information). The SST data here are averages over non-flagged neighbours in space and time, as SST is not retrieved at high rain rates.

The critical value $w_{\mathrm{c}}$ is determined by an iterative procedure with an initial guess, followed by a fit to equation (1) above $w_{\mathrm{c}}$. In Fig. 3, the curve marked 'precipitating' is computed by eliminating points explicitly flagged as non-precipitating in the microwave algorithm; the maximum near the critical point is seen despite the probable inclusion of some points with insignificant rain.

\section{Received 11 January 2006; accepted 28 April 2006; published 28 May 2006.}

\section{References}

1. Yeomans, J. Statistical Mechanics of Phase Transitions (Clarendon, Oxford, 1992).

2. Tang, C. \& Bak, P. Critical exponents and scaling relations for self-organized critical phenomena. Phys. Rev. Lett. 23, 2347-2350 (1988).

3. Sornette, D. Critical phase transitions made self-organized: A dynamical system feedback mechanism for self-organized criticality. J. Phys. I 2, 2065-2073 (1992).

4. Arakawa, A. \& Schubert, W. H. Interaction of a cumulus cloud ensemble with the large-scale environment, part I. J. Atmos. Sci. 31, 674-701 (1974).

5. Bak, P., Tang, C. \& Wiesenfeld, K. Self-organized criticality: An explanation of $1 /$ f noise. Phys. Rev. Lett. 59, 381-384 (1987)

6. Bretherton, C. S., Peters, M. E. \& Back, L. E. Relationships between water vapor path and precipitation over the tropical oceans. J. Clim. 17, 1517-1528 (2004).

7. Parsons, D. B., Yoneyama, K. \& Redelsperger, J.-L. The evolution of the tropical western Pacific ocean-atmosphere system following the arrival of a dry intrusion. Q. J. R. Meteorol. Soc. 126, $517-548(2000)$.

8. Tompkins, A. M. Organization of tropical convection in low vertical wind shears: The role of wate vapor. J. Atmos. Sci. 58, 529-545 (2001).

9. Grabowski, W.W. MJO-like coherent structures: Sensitivity simulations using the cloud-resolving convection parameterization (CRCP). J. Atmos. Sci. 60, 847-864 (2003).

10. Derbyshire, S. H. et al. Sensitivity of moist convection to environmental humidity. Q. J. R. Meteorol. Soc. 130, 3055-3079 (2005)

11. Marro, J. \& Dickman, R. Nonequilibrium Phase Transitions in Lattice Models (Cambridge Univ. Press, Cambridge, 1999).

12. Dickman, R., Vespignani, A. \& Zapperi, S. Self-organized criticality as an absorbing-state phase transition. Phys. Rev. E 57, 5095-5105 (1998).

13. Christensen, K. et al. Avalanche behavior in an absorbing state Oslo model. Phys. Rev. E 70, $067101(2004)$
14. Pruessner, G. \& Peters, O. Self-organized criticality and absorbing states: Lessons from the Ising model. Phys. Rev. E 73, 025106 (2006).

15. Xu, K.-m. \& Emanuel, K. A. Is the tropical atmosphere conditionally unstable? Mon. Weath. Rev. 117, 1471-1479 (1989).

16. Arakawa, A. The cumulus parameterization problem: Past, present, and future. J. Clim. 17, 2493-2525 (2004)

17. Emanuel, K. A., Neelin, J. D. \& Bretherton, C. S. On large-scale circulations in convecting atmospheres. Q. J. R. Meteorol. Soc. 120, 1111-1143 (1994).

18. Neelin, J. D. \& Zeng, N. A quasi-equilibrium tropical circulation model-formulation. J. Atmos. Sci. 57, 1741-1766 (2000).

19. Fisher, M. E. \& Barber, M. N. Scaling theory for finite-size effects in the critical region. Phys. Rev. Lett. 28, 1516-1519 (1972).

20. Privman, V., Hohenberg, P. C. \& Aharony, A. in Phase Transitions and Critical Phenomena Vol. 14 (eds Domb, C. \& Lebowitz, J. L.) Ch. 1, 1-134 (Academic, New York, 1991).

21. Houze, R. A. Cloud Dynamics (Academic, San Diego, 1993).

22. Stauffer, D. \& Aharony, A. Introduction to Percolation Theory 2nd edn (Taylor and Francis, London, 1992).

23. Peters, O., Hertlein, C. \& Christensen, K. A complexity view of rainfall. Phys. Rev. Lett. 88, 018701 (2002).

24. Lin, J. W.-B. \& Neelin, J. D. Toward stochastic moist convective parameterization in genera circulation models. Geophys. Res. Lett. 30, 1162 (2003).

25. Bretherton, C. S., Blossey, P. N. \& Khairoutdinov, M. An energy balance analysis of deep convective self-aggregation above uniform SST. J. Atmos. Sci. 62, 4273-4292 (2005).

26. Christensen, K. \& Moloney, N. Complexity and Criticality (Imperial College Press, London, 2005).

27. Wentz, F. J. \& Spencer, R. W. SSM/I rain retrievals within a unified all-weather ocean algorithm. J. Atmos. Sci. 56, 1613-1627 (1998).

28. Ikai, J. \& Nakamura, K. Comparison of rain rates over the ocean derived from TRMM microwave imager and precipitation radar. J. Atmos. Oceanic Technol. 20, 1709-1726 (2003).

\section{Acknowledgements}

This work was supported under National Science Foundation grant ATM-0082529 and National Oceanic and Atmospheric Administration grants NA05OAR4311134 and NA04OAR4310013 (J.D.N. and O.P.) and the US Department of Energy (W-7405-ENG-35) (O.P.). We thank D. Sornette for connecting the authors, and the Remote Sensing Systems rain team for discussion. This is Institute of Geophysics and Planetary Physics contribution no. 6289.

Correspondence and requests for materials should be addressed to O.P.

Supplementary Information accompanies this paper on www.nature.com/naturephysics.

Competing financial interests

The authors declare that they have no competing financial interests.

Reprints and permission information is available online at http://npg.nature.com/reprintsandpermissions/ 